\title{
Genotyping Sri Lankan women with polycystic ovary syndrome (PCOS): towards a novel screening tool
}

\author{
Umayal Branavan ${ }^{1}$, Kajan Muneeswaran ${ }^{2}$, Sulochana Wijesundera ${ }^{3}$, Naduviladath Vishvanath \\ Chandrasekharan ${ }^{2}$, Chandrika Wijeyaratne ${ }^{1}$
}

(Index words: PCOS, single nucleotide polymorphism, allele specific GPCR)

\begin{abstract}
Introduction: Polycystic ovary syndrome (PCOS) is the multigenic, endocrine disorder of young women. Inheritance of PCOS is likely to be oligogenic and genetic basis remains largely unknown. Screening the candidate genes of PCOS and their SNPS individually is time consuming. Hence, developing a tool that would help in screening multiple candidate genes simultaneously is essential to determine the exact genetic basis of PCOS.

Objectives: This study aimed to develop a simple and cost-effective genetic screening tool to simultaneously genotype 16 single nucleotide polymorphisms (SNPs) of PCOS.

Methods: The genetic screening tool was developed using allele specific real time quantitative PCR (ASqPCR) in 96 well PCR plate. Eight SNPs identified in our previous study as well as 8 SNPs identified from other reported studies that had a strong association in the etiology of PCOS were used to develop the tool. Samples from our previous study were reanalyzed using the developed genetic screening tool. Genetic screening tool results were validated with Sanger sequencing.

Results: Totally 10 AS-qPCR runs (160 reactions = 16SNPs*10runs) were performed using the developed tool and all except 3 genotype results agreed with Sanger sequencing. The tool showed $100 \%$ specificity and $96 \%$ sensitivity.

Conclusion: The developed genetic screening tool has excellent potential in determining the genotype of multiple SNPS of PCOS simultaneously. This tool is highly suitable for developing countries as a cost effective and accurate early genetic screening test for PCOS. Thus, provides a reliable, fast and user-friendly genotyping method facilitating a wider implication in clinical practice.
\end{abstract}

\begin{abstract}
Abbreviations
PCOS, Polycystic ovary syndrome; SNP, Single nucleotide polymorphism; AS-qPCR, Allele specific qPCR; PCR, Polymerase chain reaction; $\mathrm{mFG}$, modified FerrimanGallwey score; T2DM, Type 2 diabetes mellitus; CADMA, Competitive amplification of differentially melting amplicons; HRM, High resolution melting.
\end{abstract}

\section{Introduction}

Polycystic ovary syndrome (PCOS) is a common, heterogeneous endocrine disorder of young women. It was initially described as an association of polycystic ovaries (PCO) with amenorrhea, hirsutism and obesity in women presenting with infertility [1]. The current clinical definition recognizes PCOS as a wide spectrum of presentations consisting of a combination of any two of the three key features: oligo-amenorrohea, hyperandrogenism and polycystic ovaries, with the proviso secondary causes are excluded. Hyperandrogenism and ovarian dysfunction are the salient features of PCOS. The syndrome is associated with a broad spectrum of metabolic abnormalities linked to insulin resistance and hyperinsulinemia, abdominal obesity, hypertension and dyslipidemia that carry a high risk for developing type 2 diabetes mellitus (T2DM), cardiovascular disease and endometrial hyperplasia [2-5]. Hence, PCOS is a life-long condition manifesting from puberty with adverse reproductive and metabolic implications for the affected women [6]. The pathogenesis of PCOS is multifactorial and not clearly understood $[7,8]$. Albeit, due to the broad spectrum of symptoms with overlapping biochemical parameters of other disorders, the diagnosis of PCOS, often goes undetected until early adulthood due to the lack of

Ceylon Medical Journal 2021; 66: 129-137

DOI: http://doi.org/10.4038/cmj.v66i3.9491

${ }^{1}$ Department of Obstetrics and Gynaecology, Faculty of Medicine, University of Colombo, Sri Lanka, ${ }^{2}$ Department of Chemistry, Faculty of Science, University of Colombo, Sri Lanka, ${ }^{3}$ Department of Biochemistry and Molecular Biology, Faculty of Medicine, University of Colombo, Sri Lanka.

Correspondence: UB, e-mail: <umayal@obg.cmb.ac.Ik>. Received 05 March 2021 and revised version 05 May 2021 accepted 15 June 2021

This is an open-access article distributed under the terms of the Creative Commons Attribution License, which permits unrestricted use, distribution, and reproduction in any medium, provided the original author and source are credited. 
uniform guidelines for its diagnosis and management among the adolescent population. Although the clear cause for PCOS remains unknown, both environmental and genetic factors have been implicated. The composite of evidence suggests that several mutations or polymorphisms in candidate genes involved in steroid, gonadotropin and insulin pathways closely interact to set the stage for the development of PCOS [9].

The community prevalence of PCOS is 6.3\% among young Sri Lankan women [10] and is significantly high on an age specific basis when compared to other ethnic groups. PCOS has become a major health problem among women of reproductive age, with major metabolic implications that can cross generations.

Clinical expression of symptoms varies through the woman's life cycle and its expression in the reproductive period is noteworthy [11]. Hence, it is difficult to confirm the diagnosis of PCOS at any given phase of a woman's life. The lack of an accurate genetic diagnostic testing for PCOS is a problem that has arisen due to its heterogeneous clinical expression and phenotype. The genetic cause of the disease should be identified well before puberty. Identification of genetic risk factors which predispose to PCOS will facilitate early diagnosis, effective treatment and management of the disease from adolescence, thus reducing long term risks of early onset of type 2 diabetes mellitus, cardiovascular disease along with female infertility. However, involvement of multiple SNPs in PCOS is one of the major reasons for the delay in identifying the genetic cause of the disease. Hence, there is a need for the development of a tool that can screen multiple SNPs of PCOS simultaneously.

A study by Chen et al. (2010) identified SNP markers associated with PCOS using micro array chips and totally 45 SNPs can be genotyped by this method [12]. However, this method is not suitable for a developing country like Sri Lanka, since the cost of the probe and chip is very much high and cannot be affordable by the developing countries.

This study aimed to develop a genetic screening tool that can be affordable by the developing countries as well as involve simple techniques which can be performed locally in a reliable and cost effective manner to screen multiple candidate genes simultaneously to determine the exact genetic basis of PCOS at the early stage.

\section{Methods}

This work received approval from the Ethical Review Committee, Faculty of Medicine, University of Colombo, Sri Lanka (Protocol No EC-14-044). Consecutive women conforming to inclusion criteria were recruited from the Endocrine Clinic of the University Unit, Colombo, Sri Lanka. Diagnosis of PCOS was based on the Rotterdam criteria $[13,14]$.

\section{Sample size calculation}

The Schlesselman case control study formula was used for sample size calculation [15]. Details of sample size calculation were described in our previous reports [16-18].

\section{Recruitment of Subjects}

Inclusion criteria: Inclusion criteria were women whose symptoms manifested from adolescent years (11-19 years WHO), with all 3 diagnostic criteria present from 16-19 years of age [19]. The lower limit of age selection was based on the mean age of menarche in Sri Lanka being 13 years and leaving an allowance of two additional years for regularization of menstruation [20].

Anovular PCOS or amenorrhoea/oligomenorrhea: Anovular cycles are defined when the cycle length is more than 35 days, and the lack of demonstrable ovulation by mid cycle and luteal phase ultrasound scans, and midluteal serum progesterone [19]. Amenorrhoea - absence of menstrual periods for six months or more in a woman who has previously been menstruating. Oligomenorrhea - menstrual periods occurring at intervals of greater than 35 days, with only four to nine periods in a year.

Polycystic ovaries on ultrasound: defined by trans-vaginal or trans-abdominal ultrasound scan of ovaries, performed within the first 5 days from the onset of menstruation, and finding 24 or more follicles, measuring between 2 and 9 $\mathrm{mm}$ and/or an ovarian volume $>10 \mathrm{~cm}^{3}[13,14]$.

Hyperandrogenism: Clinical evidence of hirsutism by modified Ferriman-Gallwey score $(\mathrm{mFG}) \geq 8$, serum testosterone $(\mathrm{T})>3.5 \mathrm{nmol} / \mathrm{L}$ [19].

Exclusion criteria: Exclusion criteria included inherited disorders of IR such as Rabson-Mendenhall syndrome, Cushing syndrome, hyperprolactinaemia, untreated primary hypothyroidism, congenital adrenal hyperplasia or an androgen secreting ovarian/adrenal tumor; those taking corticosteroid, antiepileptic or antipsychotic drugs, history of hormonal contraception within the previous 6 months, pregnancy and the first postpartum year.

Control sample: Concurrently asymptomatic, normoandrogenic, normal cycling since adolescence, nonmedicated, consenting women of reproductive age in whom PCOS was objectively excluded by clinical, biochemical and ultrasound assessment, were recruited as controls. The control subjects were recruited from a single work setting where health promotion programs were conducted from 2012 (3 years before the study). Working women of similar ethnic and social background as the affected subjects were invited to participate in the study.

\section{Development of genetic screening tool using plate based real time allele specific quantitative PCR}

A genetic screening tool was developed to simultaneously determine the genotypes of 16 SNPs of PCOS by allele specific real time quantitative PCR (AS-qPCR) 
using a 96 well PCR plate. Eight SNPs identified in our previous study [16] as well as 8 SNPs identified from the literature of other reported studies from Asia that had a strong association in the etiology of PCOS were selected to develop the method (Table 1).

Designing of primers for AS-qPCR was based on a modified version of CADMA (competitive amplification of differentially melting amplicons) [21]. This method uses 3 primers: two allele specific primers and one common primer. Of the 2 allele specific primers, one primer is designed to amplify only the mutated allele (mutant primer) and the other primer amplifies only the wild-type allele (wild type primer). The common primer was designed to amplify both wild type and mutant alleles. Details of primer designing are described in our previous study [16].

The AS-qPCR was carried out using qPCR-HRM mix (5X Solis BioDyne) and the allele specific primer for each SNP was added separately into the wells along with a common primer followed by the addition of templates (Figure $1 \mathrm{a}$ and $1 \mathrm{~b}$ ). The final reaction mix for each assay consisted of $1 \mathrm{X}$ qPCR-HRM mix allele specific primer (0.5 $\mu \mathrm{M})$, common primer $(0.5 \mu \mathrm{M})$, template (20 ng) and adjusted the volume up to $10 \mu \mathrm{L}$ with deionized water.

The PCR cycling conditions for AS-qPCR was as follows: Initial denaturation $95^{\circ} \mathrm{C}$ for 15 minutes, followed by 40 cycles of denaturation $\left(95^{\circ} \mathrm{C}\right.$ for 15 seconds), annealing $\left(60^{\circ} \mathrm{C}\right.$ for 20 seconds) and extension $\left(72^{\circ} \mathrm{C}\right.$ for 20 seconds). Thereafter, high resolution melting (HRM) was performed from $50^{\circ} \mathrm{C}$ to $95^{\circ} \mathrm{C}$ with a temperature increase of $0.2^{\circ} \mathrm{C} / \mathrm{s}$ with 50 acquisitions $/{ }^{\circ} \mathrm{C}$.

Each single run consisted of a patient sample, positive control samples of respective SNPs and negative controls. Totally 10 randomly selected patient samples from our previous study were genotyped (totally 10 runs) using the screening tool. The genotype was determined by the $\mathrm{Cq}$ value of the amplification. To validate the plate based AS-qPCR method, the region flanking the polymorphic site was PCR amplified from genomic DNA using specific primers and then custom sequenced (Macrogen Inc, Korea) by Sanger sequencing method.

Table 1 depicted the 16 SNPs used for developing the screening assay. Primers used for the genetic screening tool are listed in Table 2.

\section{Determination of sensitivity and specificity of genetic screening assay}

The scientific reliability of the developed genetic tool is measured by the ‘sensitivity' and 'specificity' of the test. The following formula was used to calculate the sensitivity and specificity [22].

Sensitivity $=\frac{\text { Number of true positives }}{\text { Sumber of true negatives }+ \text { number of false negatives }}$
Specificity $=\quad \frac{\text { Number of true negatives }}{\text { Number of true negatives }+ \text { number of false positives }}$

Table 1. SNPs used for the development of genetic screening tool

\begin{tabular}{ll}
\hline SNPs of present study & SNPs from other reported studies \\
\hline FTO (rs9939609) & AR (rs5919393) [26] \\
FSHR (rs6165) & INSR (rs2059807) [27, 28] \\
FSHR (rs6166) & THADA (rs13429458) [28] \\
FSHB (rs6169) & THADA (rs12478601) [28,29] \\
INSR (rs1799817) & FSHR (rs2349415) [30,31] \\
GnRH (rs6185) & FSHR (rs2268361) [30,31] \\
LHB (rs1800447) & SHBG (rs9913778) [32] \\
LHCGR (rs2293275) & SHBG (rs6259) [32, 33]
\end{tabular}

FTO - fat mass and obesity-associated gene; FSHB - follicle stimulating hormone beta subunit; FSHR follicle-stimulating hormone receptor; INSR - insulin receptor. GnRH - gonadotropin-releasing hormone; $L H B$ - luteinizing hormone beta subunit; LHCGR - luteinizing hormone/choriogonadotropin receptor. AR - androgen receptor; THADA - thyroid adenoma associated gene; SHBG - sex hormone-binding globulin. 
Table 2. Primers of selected SNPs used for the development of genetic screening tool

\begin{tabular}{|c|c|c|}
\hline Gene & SNP & Primer Sequences $\left(5^{\prime} \rightarrow 3^{\prime}\right)$ \\
\hline FTO & rs9939609 & $\begin{array}{l}\text { F. wild type (T) - TAG GCT CCT CGC GAC TGC TGT GAA TAT T } \\
\text { F. mutant (A) - TAT GTT CAT TGC GAC TGC TGT GAA TAT A } \\
\text { Comn reverse - GAG TAA CAG AGA CTA TCC AAG TGC ATC AC } \\
\text { F. seq primer -CTG GCT CTT GAA TGA AAT AGG }\end{array}$ \\
\hline FSHB & rs6169 & $\begin{array}{l}\text { F. comn primer - GTA CCT TCA AGG AAC TGG TAT } \\
\text { R. wild type (C) - CGG GCA CTC TCA CTG TTC CG } \\
\text { R. mutant (T) - CAG GCA CTC TCA CTG TTA CA } \\
\text { R. seq primer - GCA CAG TAC AAT CAG TGC TGT CGC TGT C }\end{array}$ \\
\hline FSHR & rs6165 Thr307Ala & $\begin{array}{l}\text { F. wild type (T) - CAG AGA GAA TCT CTG AAC CCT AGT } \\
\text { F. mutant (C) - CAG AGA GGG TCT CTG AGC CCT AGC } \\
\text { Comn reverse - GGC AAG AAG TTG ATT ATA TGA CTC AG } \\
\text { F. seq primer - ACC CCA TGA TAT CTT CAC ATG GGT TGA A }\end{array}$ \\
\hline FSHR & rs6166 Asn680Ser & $\begin{array}{l}\text { F. wild type (T) - AGG GAC AAG TAT GTA AGT AGA ACC AT } \\
\text { F. mutant (C) - AGG GAC AAG TAT GTG AGT GGA ACC AC } \\
\text { Comn reverse: CTC TTC AGC TCC CAG AGT CAC CA } \\
\text { F. seq primer - CCA ATT TAC CTT AAA GGT ATG CCA }\end{array}$ \\
\hline INSR & rs1799817 & $\begin{array}{l}\text { F. comn primer - ATG TCC CAC CCC CAC TGG ACT CAC AAC } \\
\text { R. wild type (C) - TCG GTC ATG AAG GGC TTC ACC TGC CAT GAC } \\
\text { R. mutant (T) - TAA GTC ATG AAG GGC TTC ACC TGC CAT AAT } \\
\text { R. seq primer - CTC TGT GTA CGT GCC GGA CGA GTG GGA G }\end{array}$ \\
\hline GnRH & rs6185 & $\begin{array}{l}\text { F. comn primer - TGG CTG GAG CAG CCT TCC ACT CA } \\
\text { R. wild type (G) - CGC CTA GCT GGC CTT ATT CTA CTG ACG TG } \\
\text { R. mutant (C) - CTA CTA GCT GGC CTT ATT CTA CTG ACA TC } \\
\text { R. seq primer - CTG ACT CTG ACT TCC ATC TTC TGC AGG G }\end{array}$ \\
\hline$L H B$ & rs1800447 & $\begin{array}{l}\text { F. wild type (A) - ATT GCA TTG ATG GGG TGG CAA CA } \\
\text { F. mutant (G) - ATG GCA TTG ATG GGG TGG CAG CG } \\
\text { R. comn primer - ATC CAG GGA GCC GCT TCG GAC A } \\
\text { F. seq primer - CTG CCT CTG TGG GTC TGG CCC TGA GGT G }\end{array}$ \\
\hline LHCGR & rs2293275 & $\begin{array}{l}\text { F. wild type (T) - GTA TGC AAA TAC TTA CAG TGT TTT GTG AT } \\
\text { F. mutant (C) - AGC CGG CAA ATA CTT ACA GTG TTT TGT GAC } \\
\text { R. comn primer - CAA TGT GAA AGC ACA GTA AGG AAA GTG A } \\
\text { F. seq primer - CAA TTG CAA AGA AAA AAT TCC CAT TTT A }\end{array}$ \\
\hline$A R$ & rs5919393 & $\begin{array}{l}\text { F. wild type (C) - ACC ACT CAC CCT TTT TGC TTA GAT AGT GCC } \\
\text { F. mutant (T) - CTT ACT CAC CCT TTT TGC TTA GAT AGT ACT } \\
\text { Comn reverse - CTC CAA AGA GTC TGT CCA CAA TGA AAG T } \\
\text { F. seq primer - CAG GGA CCA GTG TGA GAA TG }\end{array}$ \\
\hline INSR & rs2059807 & $\begin{array}{l}\text { F. comn primer - GCA TTT TAT ACA ACC TCA CTG CAT CAG CCT } \\
\text { R. wild type (T) - GAA GTA TGT GAA TCA GAC CTC TTG CTT TTC AT } \\
\text { R. mutant (C) - TGG GTG TGT GAA TCA GAC CTC TTG CTT TTC AC } \\
\text { R. seq primer - CAA CGA CCC CAA ATC ACA G }\end{array}$ \\
\hline THADA & rs13429458 & $\begin{array}{l}\text { Comn forward - TGC TGT GCA AAG TTA GAA GAT GAA AC } \\
\text { R. wild type (T) - GAA GGC AGG GTA TAG GTG TAT GTA ATC AGT CTT } \\
\text { R. mutant (G) - TGG GGC AGG GTA TAG GTG TAT GTA ATC AGT CTG } \\
\text { R. seq primer - ACT GAC ATG TTT CCC ATC T }\end{array}$ \\
\hline
\end{tabular}

(Continued) 
F. wild type (C) - TGG CAG TCC TGC TGG TCT TGG TTA GTA CGA C F. mutant (T) - GAA CAT TCC TGC TGG TCT TGG TTA GTA CAA T Comn reverse: GTA AAG CCC GGG TCC TAA CAT TTT ATT GA

F. seq primer - TGG AGT TGG ACA GGT AAT AG

F. comn primer - ATA AAA ACA GGT GTC AGG CTG GAT TTG A R. wild type (A) - GAA CAG TGT CAC TGA ACT ACA GCC AAT TGA R. mutant (G) - TGG CAG TGT CAC TGA ACT ACA GCC AAT TGG R. seq primer - TCA ATC CTA CCC CCT GAC AG

F. wild type (C) - ACC ATG CTG TGA GAC GAA GGC ATC TTC TC F. mutant (T) - CTT ATG CTG TGA GAC GAA GGC ATC TTA TT R. comn primer - AAT GAA AGA TCT CAA TCC CAG GGC ACT F. seq primer - CAG AAT GAT ACA CTG GGT AGA

F. wild type (C) - TGG TCC ATC AGC TCT TCT CTT TTC CCT CTC F. mutant (T) - GAA TCC ATC AGC TCT TCT CTT TTC CCT ATT

R. comn primer - GAT GGT GTT CAC AGA TAA GGA AAG GAA

F. seq primer - CTG ACA TGT CCC TAC TCA GC

F. wild type (G) - TGG TTT GCA CTA CCT CCC TCT AGG AGC AG

F. mutant (A) - GAA TCT GCA CTA CCT CCC TCT AGG AGC AA

R. common - CAT TCA GGC AAA AAG AGG TGG AAG AGT

F. seq primer - AAG GGA GGC GGC ACA TTT T

F - forward; R - reverse; Comn - common; Seq - sequence

Table 3. Genetic screening tool results of $\mathbf{1 0}$ randomly selected patient samples

\begin{tabular}{lllllllllll}
\hline SNPs & \multicolumn{2}{ll}{ Patients sample } & & & & & & & \\
\hline & $\mathbf{1}$ & $\mathbf{2}$ & $\mathbf{3}$ & $\mathbf{4}$ & $\mathbf{5}$ & $\mathbf{6}$ & $\mathbf{7}$ & $\mathbf{8}$ & $\mathbf{9}$ & $\mathbf{1 0}$ \\
\hline FTO - T/A (rs9939609) & AA & AT & T T & AA & AT & T T & AT & T T & T T & AA \\
FSHR - G/A (rs6165) & GG & GG & GA & AA & GA & AA & GG & GA & GG & GA \\
FSHR - G/A (rs6166) & GG & GG & GA & AA & GA & AA & GG & GA & GG & GA \\
FSHB - C/T (rs6169) & CC & CC & CC & CC & T T & T T & CC & CC & CC & T T \\
INSR - C/T (rs1799817) & CC & CC & CC & CC & CC & CC & CC & CC & T T & CC \\
GnRH - G/C (rs6185) & GG & GG & GG & GG & GG & GG & GG & GG & GG & GG \\
LHB - T/C (rs1800447) & CC & CC & CC & CC & CC & CC & CC & CC & CC & CC \\
LHCGR - A/G (rs2293275) & GA & AA & GA & GA & GA & AA & GG & GA & GG & GA \\
AR - C/T (rs5919393) & T T & CC & T T & CC & CC & CT & T T & CC & CT & T T \\
INSR - A/G (rs2059807) & AA & AA & AG & AG & AA & AA & AA & GG & AA & AA \\
THADA - A/C (rs13429458) & AC & AA & AA & AC & CC* & AA & AA & AA & AC & CC* \\
THADA - C/T (rs12478601) & CC & CC & T T & T T & T T & CC & T T & T T & CC & CC \\
FSHR - T/C (rs2349415) & T T & T T & T T & CT & T T & T T & T T & CC & CT & T T \\
FSHR - C/T (rs2268361) & CC & CC & C T & T T & CC & CC & T T & CC & CC & CT \\
SHBG - C/T (rs9913778) & CC & CC & CC & T T* & CC & CT & CC & CC & CC & CC \\
SHBG - G/A (rs6259) & GG & GG & GG & GG & GG & GG & GG & GG & GG & GG
\end{tabular}

* Not agreed with the sequencing results 


\section{Master mis}

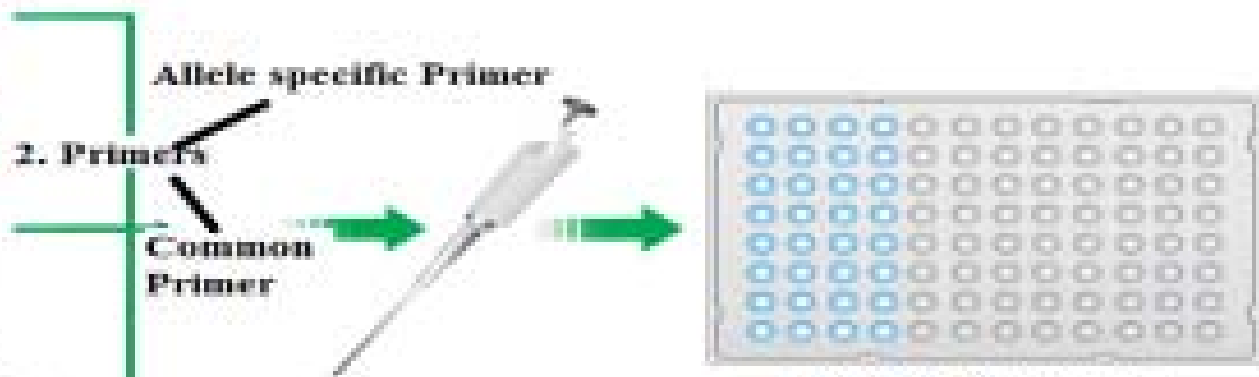

90 well PCR plate

3. Templute

Figure 1a. PCR plate based AS-qPCR.

\begin{tabular}{|c|c|c|c|c|c|c|c|c|c|c|c|}
\hline \multirow[b]{2}{*}{ C } & \multicolumn{5}{|c|}{ SNP 1} & \multicolumn{6}{|c|}{ SNP 2} \\
\hline & C & $\mathbf{P}$ & $\mathbf{P}$ & $-\mathrm{VE}$ & $-V E$ & $\mathrm{C}$ & C & $\mathbf{P}$ & $\mathbf{P}$ & $-\mathrm{VE}$ & $-\mathrm{VE}$ \\
\hline FTOT & FTOA & FTOT & FTOA & FTOT & FTOA & FSHRrs & FSHRrs & FSHRrs & FSHRr & FSHR & FSHRrs \\
\hline \multirow[t]{2}{*}{$\mathbf{T}$} & A & $\mathbf{T}$ & A & $\mathbf{T}$ & A & 6165 & 6165 & 6165 & 6165 & 6165 & 6165 \\
\hline & & & & & & $\mathbf{T}$ & C & $\mathbf{T}$ & C & $\mathbf{T}$ & C \\
\hline A1 & A 2 & A3 & A4 & A5 & A6 & A7 & A8 & A9 & A 10 & A11 & A 12 \\
\hline
\end{tabular}

Figure 1b. Schematic diagram of 1st row of 96 well PCR plate.

$\mathrm{C}$ - positive control samples; $\mathrm{P}$ - patient samples; - VE - negative control

Figure 1 b. showing the 1st row of the 96 well PCR plate and the alignment of SNPs FTO (rs9939609) and FSHR (rs6165). A1 and A2 wells - positive control samples for T and A allele of FTO rs9939609 SNP respectively. A3 and A4 wells unknown patient sample with allele specific for T and A respectively. A5 and A6 wells - negative control for T and A allele respectively. Similarly, all the other SNPs were aligned and the respective positive controls, sample and negative controls were added into the desired wells.

\section{Results}

The AS-qPCR screening tool was developed using a 96 well PCR plate. A patient sample was simultaneously genotyped for 16 SNPs in a single run. Table 3 depicts the results of 10 randomly selected patient samples genotyped for 16 SNPs using the screening tool.

The screening assay results of 10 randomly selected patient samples were validated with Sanger sequencing. Figure 2 shows the results of AS-qPCR and respective Cq values. The Cq values $<30$ indicates positive amplification of the target. If the Cq value of one primer set is $>30$, it indicates the absence of the respective allele in the sample; thereby homozygous towards the other allele. When both primer sets show Cq value $<30$, the sample is heterozygous.

With reference to the Table 3 results, sensitivity and specificity of the developed tool are calculated using following formula;

Sensitivity of the tool $=$

Number of true positives (69)

$* 100$

Number of true positives (69) + number of false negatives (3)

$=96 \%$

Specificity $=$

Number of true negatives (91)

$* 100$

Number of true negatives (91) + number of false positives (0)

$=100 \%$ 
The developed genetic tool showed $100 \%$ specificity and 96\% sensitivity. Two patients' samples genotyped for THADA (rs13429458) and one patient sample genotyped for SHBG (rs9913778) polymorphisms were detected as homozygous although they were heterozygous. There were no false positive genotype results.

\section{Discussion}

The development of biotechnology to be benchmarked as of 'state of the art' in Sri Lanka is still to be achieved. Although many initiatives have been adopted in the field, there is much more to do for achieving a satisfactory level. A major gap is the lack of advanced technology in the form of instruments (e.g. Capillary electrophoresis for Sanger sequencing) in Government institutions. Therefore, most of the Sanger sequencing tests of PCR products as well as DNA sequencing for next generation sequencing (NGS) are outsourced to overseas and require transportation charges as well as tedious documentation procedures. Hence, there is a real need to develop a simple and cost-effective genotyping method which can be performed locally to match the growing demand for rapid and reliable genotyping of medical conditions that are commonly encountered, such as PCOS.

In recent years, conventional polymerase chain reaction (PCR) techniques have been replaced by quantitative real-time PCR (qPCR). The benefits of qPCR in relation to conventional PCR include speed, reproducibility and quantitative ability. Additional operational advantages of qPCR include greater sensitivity and reproducibility, with the potential to replace conventional PCR in routine diagnostic practice. The present study developed AS-qPCR in 96 well PCR plates for genotyping multiple SNPs of PCOS which work on the principle of high-resolution melting in real time PCR instruments. Since the real time PCR instruments are currently available in most Government institutions as well as in private laboratories, the developed method can be easily performed locally without the need for advanced molecular biology techniques. It is noteworthy, that the principles of HRM and AS-qPCR have been used and recommended by some lead centers for genotyping [21, 23-25].

The cost for Sanger sequencing (Macrogen Inc, Korea) for 250 samples is 1,750 USD (7 USD per read). As the Sanger sequencing facility is not available in many institutions in Sri Lanka, samples need to be transported overseas for genotyping. Hence, the turnaround time to obtain results is minimum 5 days after DNA extraction. However, the pursued AS-PCR method can be carried out locally and the turnaround time after extraction of DNA is maximum 3 - 4 hours. Nevertheless, the developed tool eliminates the need for overseas transportation thereby reducing the overall cost and the total cost for HRM/ASPCR for 250 samples is around 150 USD. Although cost of
Sanger sequencing is low in developed countries, it is still an expensive method for a developing country like Sri Lanka.

The AS-qPCR in 96 well PCR plates provide a convenient and cost-effective method for genotyping SNPs of common polymorphisms of PCOS. Major limitation of the tool is that initially samples need to be validated by sequencing to confirm the results. However, by applying samples of known genotypes as a reference, the assay can be used for reliable genotyping of samples of unknown status.

Before using a genetic test in clinical settings, it is important to be sure that the test is valid and efficient. The validity of a screening test is based on its accuracy in identifying diseased and non-diseased persons. Validity of the genetic tool is calculated by using sensitivity and specificity. It can only be determined if the accuracy of the screening test can be compared to with a "gold standard" method. Hence, in this study genetic tool results were validated by Sanger sequencing method, which is considered as "gold standard" for validating the sequence of specific genes.

Ideally, a test should provide a high sensitivity and specificity. The developed genetic tool had the $96 \%$ sensitivity and $100 \%$ specificity which means the tool has the ability to correctly identify the patients with mutated genes and normal genes. Thus, confirmed an ideal tool for screening multiple SNPs simultaneously. Therefore, the genetic tool can be used in the clinical settings after validation.

This tool is highly suitable for the developing countries, where no advanced techniques and instruments are available. Since real-time PCR instruments are standard equipment in most molecular diagnostic laboratories, the novel method developed can be easily performed, with potential to be rated as the most cost-effective and efficient genotyping technique. Utilizing samples of known genotypes as controls, the tool can be applied for clinical settings. Additionally, this method is more feasible to use in a country like Sri Lanka, where only a few SNP variations have yet been identified in the population.

More importantly, this tool can be modified to different populations based on their occurrence of SNPs and also can be used in various diseases that are associated with multiple candidate genes. We propose that this newly developed tool has potential to pave the path towards better understanding of the causative factors and those associated with the graded risks of complications of PCOS. Overall, such an approach may help foster a better understanding of the pathophysiology of PCOS in different subgroups and populations. Such knowledge could then be leveraged to devise the most optimal screening and effective management for young women with PCOS from different subgroups and ethnicities. 
In conclusions, allele specific real time PCR are simple, fast, cost-effective and efficient genotyping techniques, feasible in many diagnostic units, as real time PCR instruments are standard equipment in most molecular diagnostic laboratories. Moreover, the developed novel genetic screening tool has excellent potential in determining the genotype of multiple SNPs of PCOS simultaneously. This tool is highly suitable for developing countries as a cost effective and accurate early genetic screening test for PCOS.

\section{Acknowledgements}

We thank the staff of the Professorial Obstetrics and Gynaecology Department and Professorial Unit of De Soysa Hospital for Women, Colombo for their ready assistance and support with ultrasound scanning, all staff of the Reproductive Biology and Endocrinology Laboratory, at the Department of Obstetrics and Gynaecology, Faculty of Medicine and the patients and their families for their wonderful cooperation. We also thank the staff of Bank of Ceylon, Head office, Colombo for their voluntary participation for the study. We specially thank Dr. Anoma Senanayake for her excellent support during the ultrasound examinations. We also thank Dr. Sumudu Jayasinghe and Dr. Dakshila Galappathi for their excellent support in coordinating the patient evaluation and sample collections.

\section{Author contributions}

The study was designed by CW, SW and NVC. Experiments were performed by UB and KM, and UB wrote the scripts and performed the statistical analysis. Writing, reviewing, and editing were done by UB, CW, SW and NVC.

\section{Declaration}

Ethics approval and consent to participate: This study was approved by the Ethics Review Committee (EC-14044), Faculty of Medicine, University of Colombo, Sri Lanka. Written informed consent was obtained from all participants.

Data access: The data generated in this study have been submitted to the Figshare repository (https:/figshare. com/articles/Complete_Gene_results_BMC_sav/ 11637264).

Funding: This study was funded by National Research Council (Grant No. 15-149) of Sri Lanka and University of Colombo (Grant no: AP/3/2/2017/SG/09).

Conflicts of interest: The authors declare that they have no competing interests.

\section{References}

1. Stein IF, Leventhal ML. Amenorrhea associated with bilateral polycystic ovaries. American Journal of Obstetrics and Gynecology 1935; 29: 181-91.

2. Dunaif A, Segal KR, Futterweit W, Dobrjansky A. Profound peripheral insulin resistance, independent of obesity, in polycystic ovary syndrome. Diabetes 1989; 38(9): 1165-74.

3. Legro RS, Kunselman AR, Dodson WC, Dunaif A. Prevalence and predictors of risk for type 2 diabetes mellitus and impaired glucose tolerance in polycystic ovary syndrome: a prospective, controlled study in 254 affected women. The Journal of Clinical Endocrinology and Metabolism 1999; 84(1): 165-9.

4. Korhonen S, Hippeläinen M, Niskanen L, Vanhala M, Saarikoski S. Relationship of the metabolic syndrome and obesity to polycystic ovary syndrome: a controlled, population-based study. American Journal of Obstetrics and Gynecology 2001; 184: 289-296.

5. Kim JJ, Choi YM. Dyslipidemia in women with polycystic ovary syndrome. Obstetrics and Gynecology Science 2013; 56: 137-142.

6. Dunaif A, Fauser BC. Renaming PCOS - a two-state solution. The Journal of Clinical Endocrinology and Metabolism 2013; 98: 4325-8.

7. Goodarzi MO, Dumesic DA, Chazenbalk G, Azziz R. Polycystic ovary syndrome: etiology, pathogenesis and diagnosis. Nature Reviews Endocrinology 2011; 7: 219-31.

8. Ehrmann DA. Polycystic ovary syndrome. The New England Journal of Medicine 2005; 352(12): 1223-36.

9. Dunaif A. Insulin resistance and the polycystic ovary syndrome: mechanism and implications for pathogenesis. Endocrine Reviews 1997; 18: 774-800.

10. Kumarapeli V, Seneviratne RA, Wijeyaratne CN, Yapa RM, Dodampahala SH. A simple screening approach for assessing community prevalence and phenotype of polycystic ovary syndrome in a semi-urban population in Sri Lanka. American Journal of Epidemiology 2008; 168(3): 321-28.

11. Fauser BC, Tarlatzism BC, Rebar RW, et al. Consensus on women's health aspects of polycystic ovary syndrome (PCOS): the Amsterdam ESHRE/ASRM-Sponsored 3rd PCOS Consensus Workshop Group. Fertility and Sterility 2012; 97(1): 28-38.e25. DOI: 10.1016/j.fertnstert. 2011.09.024.

12. Chen Z, He L, Shi Y, et al. SNPs associated with Polycystic Ovary Syndrome, chips comprising the same and the use thereof. 2010; Patent no - WO2011150545A1.

13. Rotterdam ESHRE/ASRM-Sponsored PCOS consensus workshop group. Revised 2003 consensus on diagnostic criteria and long-term health risks related to polycystic ovary syndrome. Fertility and Sterility 2004; 81: 19-25.

14. Rotterdam ESHRE/ASRM-Sponsored PCOS consensus workshop group. Revised 2003 consensus on diagnostic criteria and long-term health risks related to polycystic ovary syndrome (PCOS). Human Reproduction 2004; 19: 41-47. 
15. Schlesselman J. Case-Control Studies: Design, Conduct, Analysis. New York: Oxford University Press 1982; 354.

16. Branavan U, Muneeswaran K, Wijesundera S, Jayakody S, Chandrasekharan V, Wijeyaratne C. Identification of selected genetic polymorphisms in polycystic ovary syndrome in Sri Lankan women using low cost genotyping techniques. PLOS ONE 2018; 13(12): e0209830. https://doi.org/10.1371/journal.pone.0209830.

17. Branavan U, Muneeswaran K, Wijesundera WSS, Senanayake A, Chandrasekharan NV, Wijeyaratne CN. Association of Kiss1 and GPR54 Gene Polymorphisms with Polycystic Ovary Syndrome among Sri Lankan Women. BioMed Research International 2019. https://doi.org/10.1155/2019/6235680.

18. Umayal B, Jayakody SN, Chandrasekharan NV, Wijesundera WS, Wijeyaratne CN. Polycystic ovary syndrome (PCOS) and kisspeptin - A Sri Lankan study. J Postgrad Med. 2019; 65(1): 18-23.

19. Boteju WMM, Karunarathna GDKN, Udayangani SAD, Silva KGH, Wijeyaratne CN. Markers of hyperandrogenism in South Asians with polycystic ovary syndrome. Sri Lanka Journal of Diabetes, Endocrinology and Metabolism 2014; 4: 3-8.

20. Balasooriya S, Fernando MA. Seasonal variation of menarche in Sri Lanka. Ceylon Medical Journal 1988; 33 : 105-9.

21. Borgbo T, Sommer Kristensen L, Lindgren I, Yding Andersen C, Hansen LL. Genotyping common FSHR polymorphisms based on competitive amplification of differentially melting amplicons (CADMA). Journal of Assisted Reproduction and Genetics 2014; 31: 1427-36.

22. Boyce DA. Evaluation of Medical Laboratory Tests. Orthopaedic Physical Therapy Secrets (Third Edition): 2017; 125-34. https://doi.org/10.1016/B978-0-323-28683$1.00017-5$

23. Liew M, Pryor R, Palais R, Meadows C, Erali M, Lyon E, Wittwer C. Genotyping of single-nucleotide polymorphisms by high-resolution melting of small amplicons. Clinical Chemistry 2004; 50(7): 1156-64.

24. Siomka M, Sobalska-Kwapis M, Wachulec M, Bartosz G, Strapagiel D. High resolution melting (HRM) for highthroughput genotyping-Limitations and caveats in practical case studies. International Journal of Molecular Sciences 2017; 18(11): pii: E2316. doi: 10.3390/ijms18112316.

25. Dhas DB, Ashmi AH, Bhat BV, Parija SC, Banupriya N. Modified low cost SNP genotyping technique using cycle threshold (Ct) \& melting temperature (Tm) values in allele specific real-time PCR. Indian Journal of Medical Research 2015; 142(5): 555-62.

26. Lin LH, Baracat MC, Maciel GA, Soares JMJr, Baracat EC. Androgen receptor gene polymorphism and polycystic ovary syndrome. International Journal of Gynecology and Obstetrics 2013; 120(2): 115-18.

27. Feng C, Lv PP, Yu TT, et al. The association between polymorphism of INSR and polycystic ovary syndrome: a meta-analysis. International Journal of Molecular Sciences 2015; 16(2): 2403-25.

28. Cui L, Li G, Zhong W, et al. Polycystic ovary syndrome susceptibility single nucleotide polymorphisms in women with a single PCOS clinical feature. Human Reproduction 2015; 30(3): 732-6.

29. Bao S, Ren YC, Chen ZS, et al. THADA gene variants and polycystic ovary syndrome in a Hainan Chinese population. International Journal of Clinical and Experimental Pathology 2016; 9(11): 11883-9.

30. Du T, Duan Y, Li K, Zhao X, Ni R, Li Y, Yang D. Statistical genomic approach identifies association between FSHR polymorphisms and polycystic ovary morphology in women with polycystic ovary syndrome. Biomed Research International 2015; doi: 10.1155/2015/483726

31. Laven JSE. Follicle stimulating hormone receptor (FSHR) polymorphisms and polycystic ovary syndrome (PCOS). Frontiers in Endocrinology (Lausanne) 2019; 10: 23.

32. Wickham EP, Ewens KG, Legro RS, Dunaif A, Nestler JE, Strauss JF. Polymorphisms in the SHBG gene influence serum SHBG levels in women with polycystic ovary syndrome. The Journal of Clinical Endocrinology Metabolism 2011; 96(4): E719-27.

33. Liu Y, Zhao XX, Hu XJ, et al. Effect of sex hormonebinding globulin polymorphisms on the outcome of in vitro fertilization-embryo transfer for polycystic ovary syndrome patients: A case-control study. Journal of Cellular Biochemistry 2019; 120(3): 4675-86. 